\title{
Pigmented epithelioid melanocytoma: a rare lytic bone lesion involving intradural extension and subtotal resection in a 14-month-old girl
}

\author{
Preston D’Souza, BS, ${ }^{1}$ Erin K. Barr, MD, ${ }^{2}$ Seshadri D. Thirumala, MD, ${ }^{2}$ Roy Jacob, MD, ${ }^{3}$ and \\ Laszlo Nagy, MD²
}

${ }^{1}$ School of Medicine, ${ }^{2}$ Department of Pediatrics, and ${ }^{3}$ Department of Radiology, University Medical Center, Texas Tech University Health Sciences Center, Lubbock, Texas

\begin{abstract}
Pigmented epithelioid melanocytomas (PEMs) are low-grade, intermediate-type borderline melanocytic tumors with limited metastatic potential. To date, PEMs have been treated via gross-total resections. Postoperative recurrence and mortality are rare. This case highlights a unique presentation of a PEM that involved bone destruction and intradural infiltration, which required a subtotal resection. To the authors' knowledge, this is the first report of a PEM extending through the dura and necessitating subtotal resection, which is contrary to the standard of care, gross-total resection. Surveillance imaging 10 months after resection remained negative for clinical and radiological recurrence.

https://thejns.org/doi/abs/10.3171/2020.1.PEDS19359
\end{abstract}

KEYWORDS pigmented epithelioid melanocytoma; pediatrics; subtotal resection; oncology

$\mathrm{P}$ IGMENTED epithelioid melanocytomas (PEMs) are rare dermatological tumors that share common histological features with the epithelioid blue nevus and animal-type melanoma. ${ }^{1-3}$ Preliminary findings have shown PEM to be most prevalent in adolescents and young adults, with a few congenital cases reported in the literature. ${ }^{4-7}$ PEM most commonly occurs on the extremities, head, neck, and trunk..$^{2,7-9}$ Mortality and recurrence rates are low, with the highest recurrence rate reported in the literature at $18 \% .^{7-9} \mathrm{PEMs}$ rarely metastasize beyond regional lymph nodes, with few reports of systemic metastasis and local recurrences. ${ }^{7,8}$ PEMs are usually isolated, although they can occur as an adjunct of the Carney complex, and are not correlated with sun exposure. ${ }^{1}$ Due to their indolent nature, the standard treatment for these tumors is grosstotal resection followed by observation. Currently, PEMs are classified as low-grade, intermediate-type borderline melanocytic tumors with limited metastatic potential. ${ }^{1-3,7}$

\section{Case Report}

We present the case of a 14-month-old female patient with an expansile lesion in the occipital region.
History

The child's birth was uneventful. Two closed indentations were noticed on the scalp overlying the posterior parietal bone during the child's early infancy. Both indentations were expected to close as the child grew, but by the time she was 12 months old they had expanded, with a soft mass overlying the posterior aspect of the calvaria. By 14 months of age the patient was referred to pediatric neurosurgery. The patient had no significant clinical symptoms. Development was normal for age.

\section{Examination}

Initially, a resolving cephalohematoma was suspected due to the clinical presentation of a soft, bulging mass on top of the infant's skull, but subsequent neuroimaging revealed a soft-tissue density within the scalp that arose from the posterior parietal calvaria centrally and left of the midline, measuring $4 \times 1.5 \times 6.3 \mathrm{~cm}$, with accompanying bone destruction (Fig. 1). T1-weighted MRI displayed a hyperintense lesion (Fig. 2A) with homogeneous postcontrast enhancement (Fig. 2B and C). No other lesions were identified. There was no lymphadenopathy. Based on 

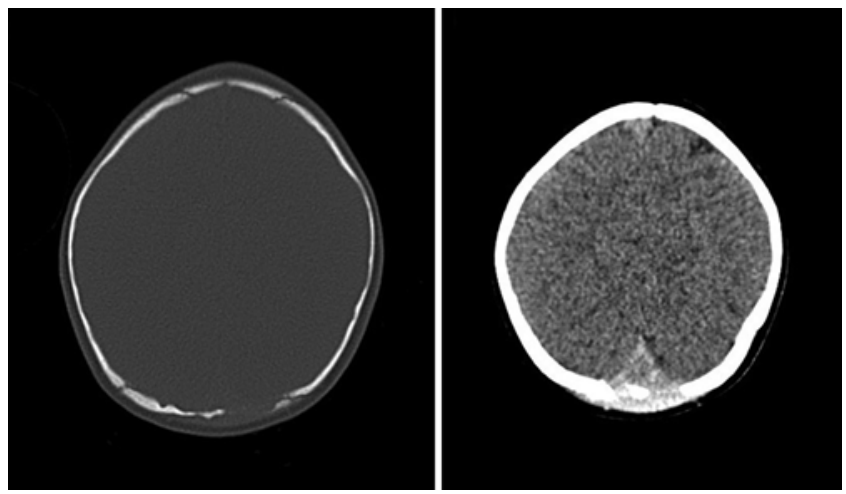

FIG. 1. Left: Axial noncontrast head CT in the bone window demonstrates a lytic lesion involving the parietal bone posteriorly. Right: Axial noncontrast head CT in the brain window demonstrates a soft-tissuedensity lytic lesion involving the parietal bone posteriorly.

these findings, a biopsy was warranted as sinus pericranii and eosinophilic granuloma were considered in the differential diagnoses.

\section{Operation and Pathological Findings}

A biopsy was performed, with pathological analysis showing the presence of cellular proliferation of mediumsized epithelioid cells with exuberant cytoplasmic coarse melanin (Fig. 3). The sample was sent for a second opinion, which confirmed the diagnosis of PEM, most likely congenital PEM given the age of the patient. An oncology consultation was undertaken, and PET scanning was performed to look for any signs of metastasis or lymph node involvement, but the tumor was non-PET avid. CT scans of the neck, chest, abdomen, and pelvis did not show any evidence of metastases. As is the standard of care, surgical resection was performed. Intraoperative exposure of the lesion revealed intradural infiltration by the tumor, which involved the wall of the superior sagittal sinus (Fig. 4 left) as well as two dural tears. Dural tears were repaired with suturing. A layer of the tumor was left on the dura due to the risk of intraoperative perforation of the superior sagittal sinus (Fig. 4 right), and the tumor layer was co- agulated. In consultation with the otolaryngology (ENT) department, cranioplasty was performed for the portions of the skull that were invaded by the tumor via plate implantation.

\section{Postoperative Course}

At the 10-month follow-up, no regrowth of the tumor was found, and at the time of this report the patient remained asymptomatic while meeting all developmental milestones. Postoperative MRI showed no evidence of recurrent or residual tumor. The sample was submitted to FoundationOne for next-generation sequencing (NGS)based genomic profiling, which revealed no targetable alterations. Testing did uncover a low tumor mutational burden and stable microsatellite status, as well as a mutation in the TNFAIP3 gene that codes for a tumor necrosis factor, A20, and regulates nuclear factor $\kappa \mathrm{B}$ signaling and apoptosis, and for which no current targeted therapies exist.

\section{Discussion}

This case highlights a unique clinical presentation of PEM, along with intraoperative characteristics and management. Even though the lesion was present since birth without causing any neurological deficits or milestone delays, the child presented to our neurosurgery department at the age of 14 months. The clinical suspicion of a resolving type 2 or calcified cephalohematoma was high due to the presence of a seemingly benign mass..$^{10,11}$ However, neuroimaging revealed an expansile lesion with destruction in the posterior parietal bone and extended the diagnostic differential to include eosinophilic granuloma and sinus pericranii. Subsequent analysis of a biopsy sample revealed medium-sized epithelioid cells with exuberant cytoplasmic coarse melanin. Histologically, PEMs are reported to contain 3 cell types: blue nevus-like dendritic cells, polygonal pigmented cells, and large epithelioid cells with large vesicular nuclei and cytoplasm. ${ }^{2}$ The patient's biopsy sample clearly showed heavy melanin pigments with epithelioid cells, confirming the diagnosis of PEM.
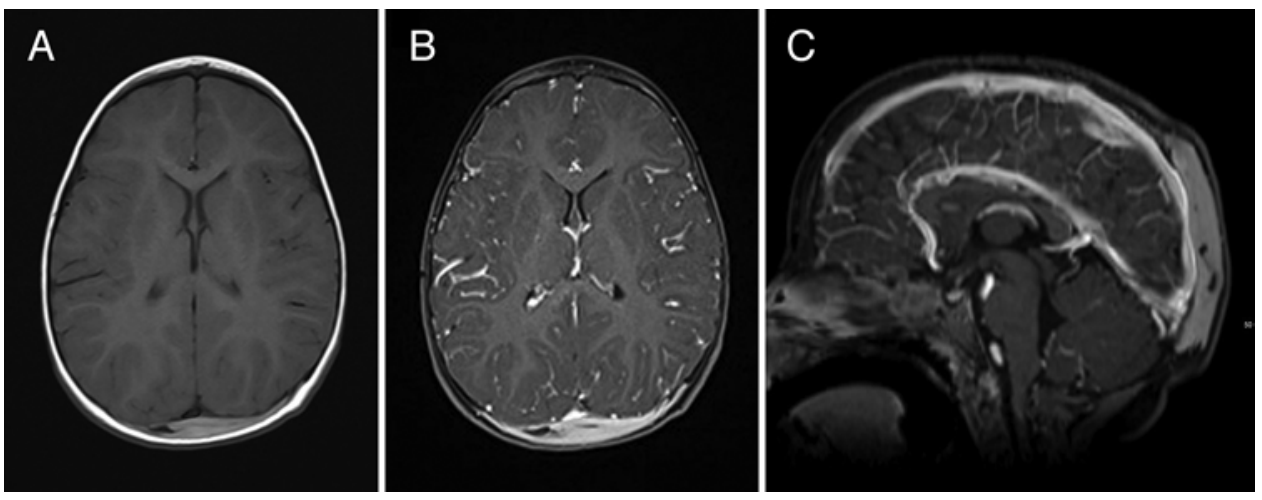

FIG. 2. A: Axial T1 precontrast imaging of the brain demonstrates a $T 1$ hyperintense soft-tissue lesion abutting the superior sagittal sinus posteriorly. B: Axial T1 postcontrast imaging of the brain demonstrates avid enhancement of the soft-tissue mass abutting the superior sagittal sinus. C: Sagittal T1 postcontrast MRI of the brain demonstrates an avidly enhancing mass involving the posterior parietal calvaria and abutting the superior sagittal sinus. 


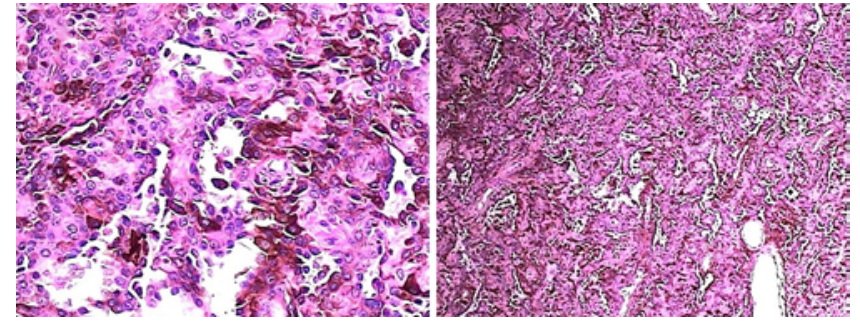

FIG. 3. H \& E staining reveals heavy melanin pigments with epithelioid cells; original magnification $\times 200$ (left) and $\times 100$ (right). Figure is available in color online only.

The head and neck are common locations for PEMs.,5,79,12 Our patient's PEM was beneath the scalp, involved erosion of the posterior parietal bone, and extended through the dura. There has been one other reported case in a newborn of a congenital subcutaneous PEM that included erosion of the occipital bone and involvement of the dura, but unlike the present case, there was no extension through the dura and therefore treatment with gross-total resection was performed. ${ }^{4}$

With complete resection of the mass, outcomes for pediatric PEMs are usually ideal and consistent with their indolent nature. ${ }^{7}$ Outcomes and clinical course when the tumor cannot be completely excised, such as in our patient, are unknown. There are also no known chemotherapy or alternative therapeutic strategies that have known benefit in treating PEMs. The standard of care treatment is gross-total surgical resection. FoundationOne NGS testing in our case identified a mutation in the TNFAIP3 gene; however, there is no reported association between this gene and PEMs, nor do chemotherapy agents exist that target this gene. This mutation is therefore of unknown clinical significance. For this reason, and with no evidence of metastases, the decision was made to watch our patient and perform follow-up MRI every 3 months for the first year after subtotal resection. If the PEM recurs, then the patient will undergo an extensive operation to remove the PEM-infiltrated portion of dura and a subsequent duraplasty. Depending on the extent of recurrence, if distant metastases have occurred, the possibility of chemotherapy may be discussed; however, any therapy used would be experimental and of unknown benefit.

At the time of this report, the patient was 10 months postsurgery and meeting all developmental milestones, while showing no signs of disease progression or recurrence on scans.

\section{Conclusions}

PEMs are rare tumors that have low malignant potential and rarely metastasize beyond the regional lymph nodes. The present case entails a unique presentation of PEM involving bone destruction and intradural infiltration in a 14-month-old girl. Although gross-total resection is the standard of care, a subtotal resection was necessitated by the intradural infiltration. Despite being treated with subtotal rather than gross-total resection, at the time of this report the patient remained well and recurrence free at 23 months of age, which suggests that given the indolent
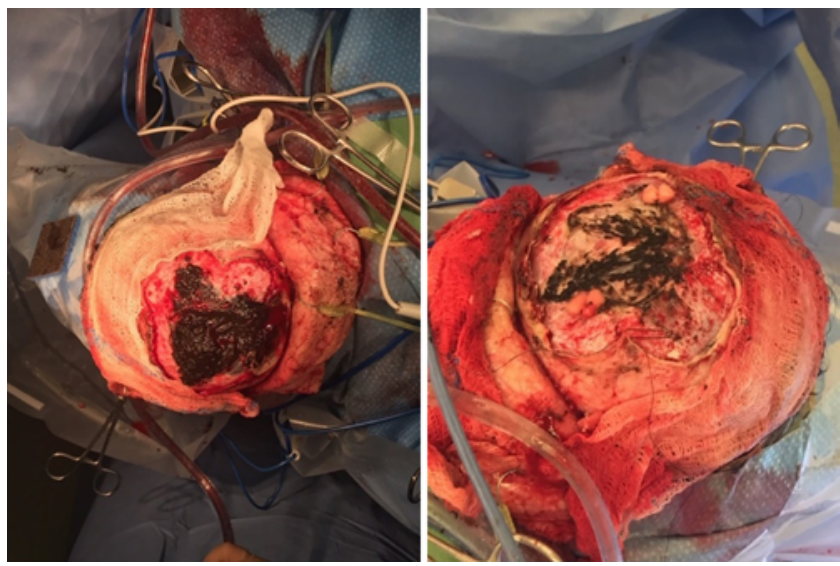

FIG. 4. Left: Intraoperative photograph showing the posterior parietal region of the skull with initial presentation of the PEM before resection. Right: Intraoperative photograph showing the posterior parietal region of the skull, with a layer of PEM retained on the dura after subtotal resection to avoid puncture of the superior sagittal sinus. Figure is available in color online only.

nature of these tumors, patients unable to receive a grosstotal resection may still have the potential for favorable outcomes.

\section{Acknowledgments}

We acknowledge the AmeriPath-Covenant Medical Center for providing us with the histopathology images.

\section{References}

1. Zembowicz A, Carney JA, Mihm MC. Pigmented epithelioid melanocytoma: a low-grade melanocytic tumor with metastatic potential indistinguishable from animal-type melanoma and epithelioid blue nevus. Am J Surg Pathol. 2004;28(1):31-40.

2. Zembowicz A, Knoepp SM, Bei T, et al. Loss of expression of protein kinase a regulatory subunit lalpha in pigmented epithelioid melanocytoma but not in melanoma or other melanocytic lesions. Am J Surg Pathol. 2007;31(11):1764-1775.

3. Zembowicz A, Scolyer RA. Nevus/melanocytoma/melanoma: an emerging paradigm for classification of melanocytic neoplasms? Arch Pathol Lab Med. 2011;135(3):300-306.

4. Battistella M, Prochazkova-Carlotti M, Berrebi D, et al. Two congenital cases of pigmented epithelioid melanocytoma studied by fluorescent in situ hybridization for melanocytic tumors: case reports and review of these recent topics. Dermatology. 2010;221(2):97-106.

5. Cohen JN, Joseph NM, North JP, et al. Genomic analysis of pigmented epithelioid melanocytomas reveals recurrent alterations in PRKAR1A, and PRKCA genes. Am J Surg Pathol. 2017;41(10):1333-1346.

6. Johnson ER, Korchak ME, Goodglick TA, et al. Pigmented epithelioid melanocytoma of the cheek, orbit, and intracranial cavity: a case report. Ocul Oncol Pathol. 2017;4(1):57-60.

7. Mandal RV, Murali R, Lundquist KF, et al. Pigmented epithelioid melanocytoma: favorable outcome after 5-year follow-up. Am J Surg Pathol. 2009;33(12):1778-1782.

8. Cheng P-S, Chuang S-S, Kuo T-T, Lai F-J. Pigmented epithelioid melanocytoma: report of a case and review of 173 cases in the literature. Dermatol Sinica. 2012;30:57-61.

9. Isales MC, Mohan LS, Quan VL, et al. Distinct genomic patterns in pigmented epithelioid melanocytoma: a molecu- 
lar and histologic analysis of 16 cases. Am J Surg Pathol. 2019;43(4):480-488.

10. Raines DA, Jain S. Cephalohematoma. Treasure Island, FL: StatPearls Publishing; 2019.

11. Wong CH, Foo CL, Seow WT. Calcified cephalohematoma: classification, indications for surgery and techniques. $\mathrm{J} \mathrm{Cra-}$ niofac Surg. 2006;17(5):970-979.

12. Howard B, Ragsdale B, Lundquist K. Pigmented epithelioid melanocytoma: two case reports. Dermatol Online $J$. 2005;11(2):1.

\section{Disclosures}

The authors report no conflict of interest concerning the materials or methods used in this study or the findings specified in this paper.

\section{Author Contributions}

Conception and design: D'Souza, Nagy. Acquisition of data: D'Souza, Thirumala. Analysis and interpretation of data: D’Souza, Jacob. Drafting the article: D'Souza, Barr. Critically revising the article: Barr, Jacob. Reviewed submitted version of manuscript: D'Souza. Approved the final version of the manuscript on behalf of all authors: D'Souza.

\section{Correspondence}

Preston D'Souza: Texas Tech University Health Sciences Center School of Medicine, Lubbock, TX.pd3003@gmail.com. 